\title{
Variable C/P composition of organic production and its effect on ocean carbon storage in glacial model simulations
}

Malin Ödalen $^{1}$, Jonas Nycander ${ }^{1}$, Andy Ridgwell ${ }^{2,3}$, Kevin I. C. Oliver ${ }^{4}$, Carlye D. Peterson ${ }^{2}$, and Johan Nilsson ${ }^{1}$

${ }^{1}$ Department of Meteorology, Bolin Centre for Climate Research, Stockholm University, 10691 Stockholm, Sweden

${ }^{2}$ Department of Earth Sciences, University of California-Riverside, Riverside, CA 92521, USA

${ }^{3}$ School of Geographical Sciences, Bristol University, Bristol BS8 1SS, UK

${ }^{4}$ National Oceanography Centre, Southampton, University of Southampton, Southampton SO14 3ZH, United Kingdom

Correspondence: Malin Ödalen (malin.odalen@misu.su.se) 


\section{S Supplementary material}

This document includes supplementary figures and tables, containing data that is described or shown in other forms in the manuscript.

In Fig. S.1, the locations of core sites for observed benthic $\delta^{13} C$ are indicated, and Holocene (HOL) observed benthic $\delta^{13} C$

5 is compared to the bottom water ocean distribution of $\delta^{13} C$ for $C t r l_{R E D}$. The positions of observation sites are the same as in Peterson et al. (2014).

For comparison with Fig. 3a, we show the surface $\mathrm{PO}_{4}$ concentration $(\mu M)$ of the control state with fixed Redfield stoichiometry $\left(C t r l_{R E D}\right.$ (Fig. S.2).

For comparison with Fig. 5, which shows model ocean $\delta^{13} C$ of model version $R E D$ compared to observations, we show 10 the correpsonding ocean sections for model version GAM (Fig. S.3).

The supplementary tables S.1 and S.2 list diagnostic variables for climate (Table S.1, model versions $R E D$ and GAM, all ensemble members), carbon and nutrients (Table S.2, all model versions, all ensemble members). Table S.3 lists model-data comparison statistics for the $C t r l$ and $G L c o m b$ simulations in each of the model versions $R E D$ and $G A M$, and for the two benthic $\delta^{13} C$ data time slices HOL (0-6 ka) and LGM (19-23 ka). 


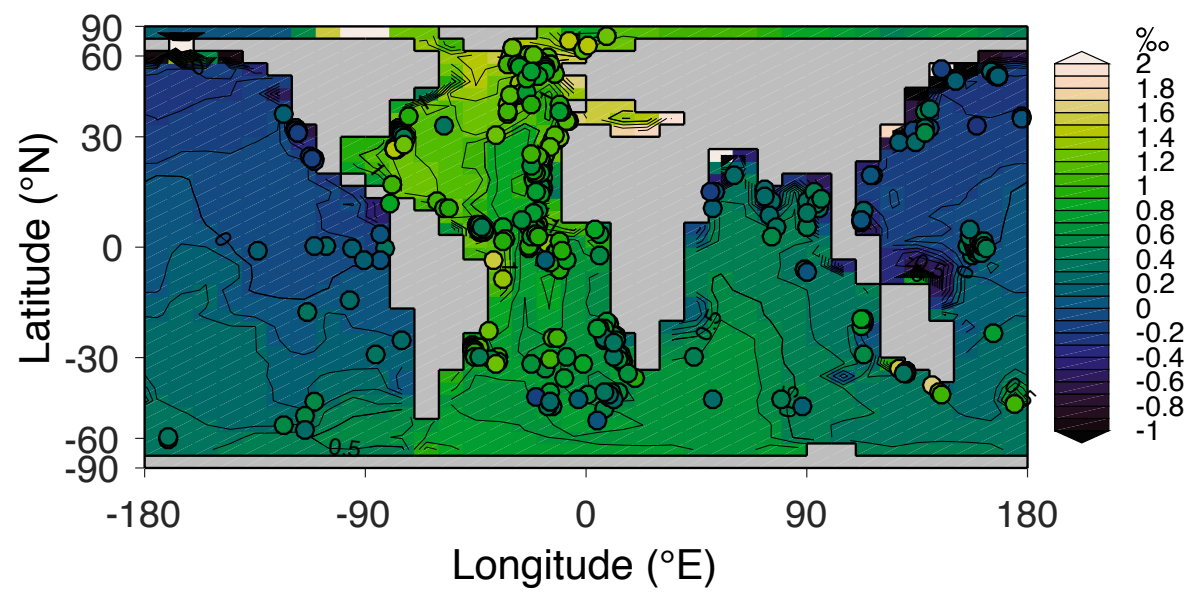

Figure S.1. $C t r l_{R E D}$ bottom water ocean $\delta^{13} C$ (contours) compared to the Holocene (HOL) time slice of observed benthic $\delta^{13} C$ (circles) of Peterson et al. (2014). 


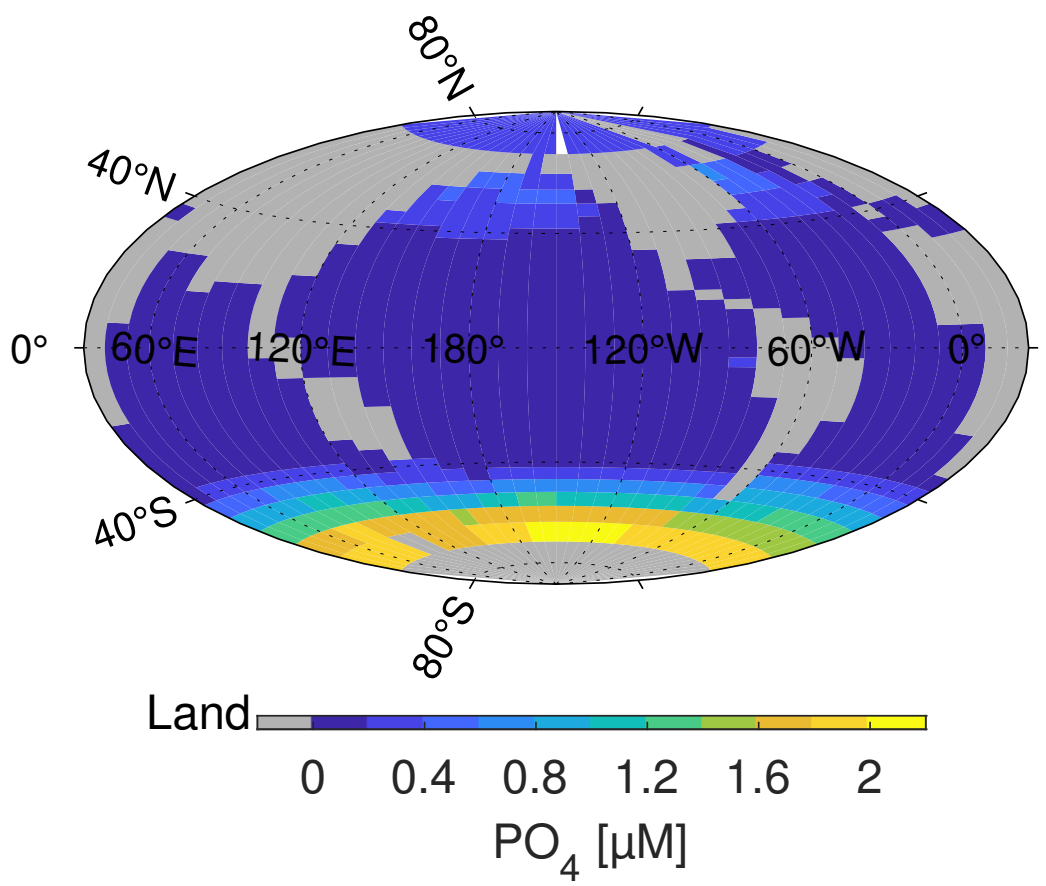

Figure S.2. Surface $\mathrm{PO}_{4}$ concentration $(\mu M)$ of the control state with fixed Redfield stoichiometry $\left(C \operatorname{trl} l_{R E D}\right)$. 

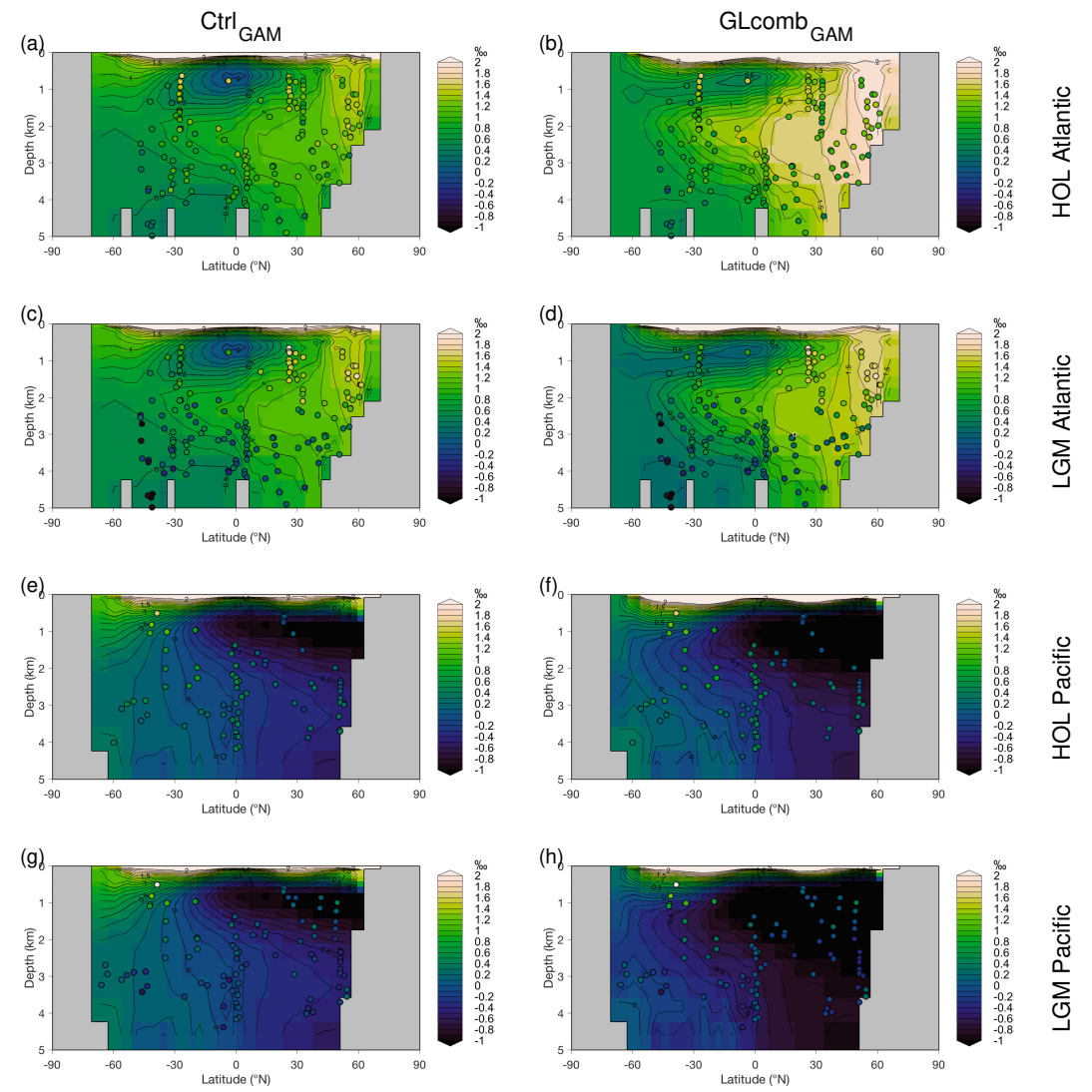

Figure S.3. Model ocean $\delta^{13} C$ (contours) compared to the two time slices (HOL and LGM) of observed benthic $\delta^{13} C$ (circles) of Peterson et al. (2014). Left hand column shows $C t r l_{G A M}$ (panels a, c, e, g) and right hand column shows $G L c o m b_{G A M}$ (panels b, d, f, h). The rows show, from top to bottom, a-b) HOL Atlantic, c-d) LGM Atlantic, e-f) HOL Pacific, g-h) LGM Pacific. Note that, when we compare $G L c o m b_{G A M}$ to LGM observations (panels d and h), a constant of $0.32 \%$ is subtracted from $G L c o m b_{G A M}$ data, to account for terrestrial release of $\delta^{13} C$-depleted terrestrial carbon. 
Table S.1. Carbon and nutrient variables in control states $(C t r l)$ and ensemble members (Table 1) of model versions $R E D$ and $G A M$. The columns list global ocean values for $\mathrm{C} / \mathrm{P}$ ratio in the export flux of organic matter (dimensionless quantity), total content of DIC (DIC $C_{c o n t}$,

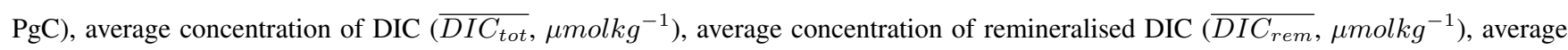
concentration of remineralised acidic carbon $\left(\overline{A C_{r e m}}, \mu m o l k g^{-1}\right)$, average concentration of $\mathrm{PO}_{4}\left(\overline{P_{t o t}}, \mu \mathrm{molkg}{ }^{-1}\right)$, average concentration of remineralised $\mathrm{PO}_{4}\left(\overline{P_{r e m}}, \mu m o l k g^{-1}\right)$ and average of the nutrient utilisation efficiency $\left(\overline{P^{*}}\right)$. The modern ocean observed C/P is given as determined by Anderson and Sarmiento (1994) and the global ocean carbon inventory (DIC cont) is estimated to $38,700 \mathrm{PgC}($ Stocker, 2014). The observational estimate for $\overline{P^{*}}$ is given by Ito and Follows (2005).

\begin{tabular}{|c|c|c|c|c|c|c|c|c|}
\hline $\begin{array}{l}\text { Experiment } \\
\text { acronym }\end{array}$ & $\begin{array}{l}\mathrm{C} / \mathrm{P} \\
\text { ratio }\end{array}$ & $\begin{array}{l}D I C_{\text {cont }} \\
(\mathrm{PgC})\end{array}$ & $\begin{array}{l}\overline{D I C_{t o t}} \\
\left(\mu \mathrm{molkg} g^{-1}\right)\end{array}$ & $\begin{array}{l}\overline{D I C_{\text {rem }}} \\
\left(\mu \mathrm{molkg}{ }^{-1}\right)\end{array}$ & $\begin{array}{l}\overline{A C_{r e m}} \\
\left(\mu m o l k g^{-1}\right)\end{array}$ & $\begin{array}{l}\overline{P_{t o t}} \\
\left(\mu m o l k g^{-1}\right)\end{array}$ & $\begin{array}{l}\overline{P_{r e m}} \\
\left(\mu m o l k g^{-1}\right)\end{array}$ & $\begin{array}{l}\text { Global } \\
\overline{P^{*}}\end{array}$ \\
\hline $\operatorname{Ctrl}_{R E D}$ & 106.00 & 35,882 & $2,234.0$ & 146.16 & 106.64 & 2.1520 & 0.9354 & 0.435 \\
\hline$C t r l_{G A M}$ & 120.85 & 36,134 & $2,249.7$ & 172.28 & 124.17 & 2.1518 & 0.9877 & 0.459 \\
\hline$L G M r f_{R E D}$ & 106.0 & 35,910 & $2,235.7$ & 136.32 & 99.982 & 2.1520 & 0.8783 & 0.408 \\
\hline$L G M r f_{G A M}$ & 120.90 & 36,162 & $2,251.4$ & 160.56 & 116.22 & 2.1518 & 0.9120 & 0.424 \\
\hline$L G M a l b_{R E D}$ & 106.00 & 35,937 & $2,237.4$ & 121.72 & 89.678 & 2.1522 & 0.7875 & 0.366 \\
\hline$L G M a l b_{G A M}$ & 121.15 & 36,192 & $2,253.3$ & 143.32 & 104.12 & 2.1520 & 0.8045 & 0.374 \\
\hline$W N S \times 0.5_{R E D}$ & 106.00 & 35,911 & $2,235.8$ & 170.91 & 125.16 & 2.1524 & 1.0983 & 0.510 \\
\hline$W N S \times 0.5_{G A M}$ & 124.75 & 36,170 & $2,251.9$ & 201.96 & 145.91 & 2.1523 & 1.1257 & 0.523 \\
\hline$R L S \times 0.75_{R E D}$ & 106.00 & 35,843 & $2,231.5$ & 141.81 & 96.857 & 2.1508 & 0.8525 & 0.396 \\
\hline$R L S \times 0.75_{G A M}$ & 118.86 & 36,084 & $2,246.5$ & 164.24 & 111.20 & 2.1505 & 0.9068 & 0.422 \\
\hline$R L S \times 1.25_{R E D}$ & 106.00 & 35,914 & $2,235.9$ & 149.20 & 114.68 & 2.1526 & 1.0069 & 0.468 \\
\hline$R L S \times 1.25_{G A M}$ & 122.69 & 36,175 & $2,252.2$ & 177.94 & 134.76 & 2.1525 & 1.0534 & 0.455 \\
\hline
\end{tabular}


Table S.1. Continued.

\begin{tabular}{|c|c|c|c|c|c|c|c|c|}
\hline $\begin{array}{l}\text { Experiment } \\
\text { acronym }\end{array}$ & $\begin{array}{l}\mathrm{C} / \mathrm{P} \\
\text { ratio }\end{array}$ & $\begin{array}{l}D I C_{\text {cont }} \\
(\mathrm{PgC})\end{array}$ & $\begin{array}{l}\overline{D I C_{t o t}} \\
\left(\mu m o l k g^{-1}\right)\end{array}$ & $\begin{array}{l}\overline{D I C_{\text {rem }}} \\
\left(\mu m o l k g^{-1}\right)\end{array}$ & $\begin{array}{l}\overline{A C_{r e m}} \\
\left(\mu m o l k g^{-1}\right)\end{array}$ & $\begin{array}{l}\overline{P_{t o t}} \\
\left(\mu m o l k g^{-1}\right)\end{array}$ & $\begin{array}{l}\overline{P_{\text {rem }}} \\
\left(\mu m o l k g^{-1}\right)\end{array}$ & $\begin{array}{l}\text { Global } \\
\overline{P^{*}}\end{array}$ \\
\hline$L_{G M d u s t_{R E D}}$ & 106.00 & 35,916 & $2,236.1$ & 113.33 & 113.02 & 2.1515 & 0.9931 & 0.462 \\
\hline$L_{G M d u s t}{ }_{G A M}$ & 124.05 & 36,179 & $2,252.4$ & 134.11 & 134.75 & 2.1512 & 1.0482 & 0.487 \\
\hline$A c o m b_{R E D}$ & 106.00 & 36,002 & $2,241.5$ & 122.01 & 94.115 & 2.1528 & 0.8259 & 0.384 \\
\hline$A_{c o m b} A M$ & 125.61 & 36,270 & $2,258.2$ & 150.58 & 113.85 & 2.1525 & 0.8452 & 0.393 \\
\hline$G L c o m b_{R E D}$ & 106.00 & 36,021 & $2,242.7$ & 146.62 & 111.86 & 2.1537 & 0.9808 & 0.455 \\
\hline$G L c o m b_{G A M}$ & 133.77 & 36,307 & $2,260.5$ & 186.19 & 138.32 & 2.1534 & 0.9813 & 0.456 \\
\hline
\end{tabular}


Table S.2. Climatic variables for all control states $(C t r l)$ and ensemble members (Table 1). The variables are atmospheric $C \mathrm{O}_{2}\left(p C \mathrm{O}_{2}^{a t m}\right.$, $\mathrm{ppm})$, global averages of temperature in the ocean $\left(T_{a v g}^{o c e},{ }^{\circ} \mathrm{C}\right)$ and in the atmosphere $\left(T_{\text {avg }}^{a t m},{ }^{\circ} \mathrm{C}\right)$, transport in the Atlantic meridional overturning circulation (AMOC, Sv) and sea ice cover (\%). The observational estimate for $\overline{T_{\text {oce }}}$ has been calculated using the World Ocean Atlas 2013 (Locarnini et al., 2013). Average modern day AMOC strength is estimated by McCarthy et al. (2015) from the RAPID-MOCHA array at $26^{\circ} \mathrm{N}$. Modern day sea ice cover is given as an interval due to seasonal variability (Comiso, 2008). No observational estimate for $\overline{T_{a t m}}$ is given, since modern atmospheric observations are strongly influenced by rising $p C O_{2}^{a t m}$ (Stocker, 2014).

\begin{tabular}{|c|c|c|c|c|c|}
\hline $\begin{array}{l}\text { Experiment } \\
\text { acronym }\end{array}$ & $\begin{array}{l}p C O_{2}^{a t m} \\
(\mathrm{ppm})\end{array}$ & $\begin{array}{l}\overline{T_{o c e}} \\
\left({ }^{\circ} \mathrm{C}\right)\end{array}$ & $\begin{array}{l}\overline{T_{a t m}} \\
\left({ }^{\circ} \mathrm{C}\right)\end{array}$ & $\begin{array}{l}\text { AMOC } \\
\text { (Sv) }\end{array}$ & $\begin{array}{l}\text { Sea ice } \\
(\%)\end{array}$ \\
\hline Observ. & 278 & 3.49 & N/A & $17.2 \pm 0.9$ & 3 to 6 \\
\hline$C t r l_{R E D}$ & 278.0 & 3.56 & 12.38 & 14.25 & 5.41 \\
\hline$C t r l_{G A M}$ & 278.0 & 3.57 & 12.38 & 14.27 & 5.42 \\
\hline$C t r l_{121}$ & 278.0 & 3.59 & 12.37 & 14.25 & 5.42 \\
\hline$L G M r f_{R E D}$ & 265.2 & 2.89 & 10.46 & 14.99 & 7.38 \\
\hline$L G M r f_{G A M}$ & 265.5 & 2.90 & 10.46 & 14.97 & 7.35 \\
\hline$L G M a l b_{R E D}$ & 252.8 & 1.97 & 8.18 & 13.82 & 9.30 \\
\hline$L G M a l b_{G A M}$ & 251.6 & 1.97 & 8.16 & 13.82 & 9.38 \\
\hline$L G M p h y_{R E D}$ & 245.4 & 1.45 & 6.83 & 13.67 & 10.6 \\
\hline$L G M p h y_{G A M}$ & 244.9 & 1.45 & 6.84 & 13.66 & 10.6 \\
\hline$W N S \times 0.5_{R E D}$ & 265.1 & 3.98 & 12.12 & 12.66 & 5.68 \\
\hline$W N S \times 0.5_{G A M}$ & 261.7 & 3.99 & 12.05 & 12.72 & 5.74 \\
\hline$W N S \times 0.5_{121}$ & 263.5 & 3.97 & 12.09 & 12.66 & 5.69 \\
\hline$R L S \times 0.75_{R E D}$ & 295.8 & 3.64 & 12.66 & 14.13 & 5.21 \\
\hline$R L S \times 0.75_{G A M}$ & 301.4 & 3.68 & 12.75 & 14.09 & 5.08 \\
\hline$R L S \times 0.75_{121}$ & 298.7 & 3.65 & 12.71 & 14.13 & 5.14 \\
\hline$R L S \times 1.25_{R E D}$ & 264.0 & 3.50 & 12.13 & 14.44 & 5.68 \\
\hline$R L S \times 1.25_{G A M}$ & 262.3 & 3.49 & 12.09 & 14.46 & 5.74 \\
\hline$R L S \times 1.25_{121}$ & 259.8 & 3.49 & 12.05 & 14.46 & 5.78 \\
\hline$R L S \times 1.75_{R E D}$ & 244.7 & 3.38 & 11.77 & 14.55 & 6.06 \\
\hline$R L S \times 1.75_{G A M}$ & 234.7 & 3.33 & 11.57 & 14.56 & 6.27 \\
\hline$R L S \times 1.75_{121}$ & 240.6 & 3.36 & 11.69 & 14.59 & 6.14 \\
\hline
\end{tabular}


Table S.2. Continued.

\begin{tabular}{|c|c|c|c|c|c|}
\hline $\begin{array}{l}\text { Experiment } \\
\text { acronym }\end{array}$ & $\begin{array}{l}p C O_{2}^{a t m} \\
(\mathrm{ppm})\end{array}$ & $\begin{array}{l}T_{a v g}^{o c e} \\
\left({ }^{\circ} \mathrm{C}\right)\end{array}$ & $\begin{array}{l}T_{\text {avg }}^{\text {atm }} \\
\left({ }^{\circ} \mathrm{C}\right)\end{array}$ & $\begin{array}{l}\text { AMOC } \\
(\mathrm{Sv})\end{array}$ & $\begin{array}{l}\text { Sea ice } \\
(\%)\end{array}$ \\
\hline$L_{G M d u s t_{R E D}}$ & 262.2 & 3.48 & 12.09 & 14.44 & 5.80 \\
\hline LGMdust $_{G A M}$ & 256.9 & 3.48 & 11.99 & 14.47 & 5.87 \\
\hline LGMdust $_{121}$ & 259.5 & 3.48 & 12.05 & 14.47 & 5.78 \\
\hline$A c o m b_{R E D}$ & 222.5 & 1.45 & 6.83 & 13.67 & 10.6 \\
\hline$A c o m b_{G A M}$ & 214.9 & 1.45 & 6.84 & 13.66 & 10.6 \\
\hline$A c o m b_{121}$ & 217.3 & 1.44 & 6.84 & 13.59 & 10.6 \\
\hline$G L c o m b_{R E D}$ & 214.9 & 1.94 & 6.86 & 12.05 & 10.2 \\
\hline $\operatorname{GLcomb}_{G A M}$ & 198.2 & 1.91 & 6.85 & 12.09 & 10.3 \\
\hline GLcomb $b_{121}$ & 206.2 & 1.93 & 6.86 & 12.02 & 10.3 \\
\hline
\end{tabular}


Table S.3. Model-data comparison statistics for the Ctrl and GLcomb simulations in each of the model versions $R E D$ and $G A M$, and for the two benthic $\delta^{13} C$ data time slices HOL (0-6 ka) and LGM (19-23 ka). Correlations are computed between benthic observations and model water mass $\delta^{13} C$ in the grid cell corresponding to the observation depth. Standard deviations (STD) are the normalized values $\left(S T D_{\text {model }} / S T D_{\text {data }}\right)$, which are scaled by the number of observations.

\begin{tabular}{lllllll}
\hline Model - data & $\begin{array}{l}\text { Global } \\
\text { corr. }\end{array}$ & $\begin{array}{l}\text { Global } \\
\text { STD }\end{array}$ & $\begin{array}{l}\text { Atlantic } \\
\text { corr. }\end{array}$ & $\begin{array}{l}\text { Atlantic } \\
\text { STD }\end{array}$ & $\begin{array}{l}\text { Indo-Pacific } \\
\text { corr. }\end{array}$ & $\begin{array}{l}\text { Indo-Pacific } \\
\text { STD }\end{array}$ \\
\hline Ctrl $_{R E D}-\mathrm{HOL}$ & 0.78 & 1.23 & 0.50 & 0.83 & 0.39 & 0.81 \\
$C \operatorname{trl} l_{G A M}-\mathrm{HOL}$ & 0.76 & 1.38 & 0.45 & 0.94 & 0.36 & 0.95 \\
$G L c o m b_{R E D}-\mathrm{HOL}$ & 0.76 & 1.82 & 0.46 & 1.22 & 0.34 & 1.10 \\
$G L c o m b_{G A M}-\mathrm{HOL}$ & 0.76 & 2.11 & 0.44 & 1.46 & 0.33 & 1.31 \\
\hline $\operatorname{Ctrl}_{R E D}-\mathrm{LGM}$ & 0.58 & 0.96 & 0.64 & 0.43 & 0.06 & 0.83 \\
$C t r l_{G A M}-\mathrm{LGM}$ & 0.55 & 1.08 & 0.58 & 0.48 & 0.01 & 0.96 \\
$G L c o m b_{R E D}-\mathrm{LGM}$ & 0.58 & 1.40 & 0.61 & 0.62 & 0.06 & 1.07 \\
$G L c o m b_{G A M}-\mathrm{LGM}$ & 0.57 & 1.62 & 0.60 & 0.74 & 0.02 & 1.28 \\
\hline
\end{tabular}




\section{References}

Anderson, L. A. and Sarmiento, J. L.: Redfield ratios of remineralization determined by nutrient data analysis, Global biogeochemical cycles, 8, 65-80, 1994.

Comiso, J. C.: Variability and trends of the global sea ice cover, in: Sea ice: an introduction to its physics, chemistry, biology and geology, edited by Thomas, D. N. and Dieckmann, G. S., John Wiley \& Sons, 2008.

Ito, T. and Follows, M. J.: Preformed phosphate, soft tissue pump and atmospheric $\mathrm{CO}_{2}$, Journal of Marine Research, 63, 813-839, 2005.

Locarnini, R. A., Mishonov, A. V., Antonov, J. I., Boyer, T. P., Garcia, H. E., Baranova, O. K., Zweng, M. M., Paver, C. R., Reagan, J. R., Johnson, D. R., Hamilton, M., and Seidov, D.: World Ocean Atlas 2013, Volume 1: Temperature, Tech. Rep. 73, NOAA Atlas NESDIS, 40 pp., 2013.

10 McCarthy, G., Smeed, D., Johns, W. E., Frajka-Williams, E., Moat, B., Rayner, D., Baringer, M., Meinen, C., Collins, J., and Bryden, H.: Measuring the Atlantic meridional overturning circulation at 26 N, Progress in Oceanography, 130, 91-111, 2015.

Peterson, C. D., Lisiecki, L. E., and Stern, J. V.: Deglacial whole-ocean $\delta 13 \mathrm{C}$ change estimated from 480 benthic foraminiferal records, Paleoceanography, 29, 549-563, 2014.

Stocker, T.: Climate change 2013: the physical science basis: Working Group I contribution to the Fifth assessment report of the Intergovernmental Panel on Climate Change, Cambridge University Press, 2014. 